\section{Shielded colleagues}

Sir, in mid-March when the lockdown began I was contacted by the NHS and notified that due to my diagnosis of Crohn's disease I had been placed in the highly vulnerable category and thus was advised to shield for 12 weeks.

Whilst the CDO issued guidance on 28 May 2020 that dentists will return to work on the 8 June in England there has been no guidance on how this applies to those, such as myself, who are in the shielded category.

The initial 12-week period of shielding is set to finish at the end of June, however, there are now suggestions that this will be extended further. Despite this, there has been no clarification as to whether this applies to all shielded individuals or whether this should be decided on a case by case basis on the advice of one's doctor (my own medical team have informed me they are unsure of how the shielding rules apply to dentists and have not received any further guidance on this matter to date).

As a general dental practitioner, I am anxious to get back to work and to provide a much-needed service to my patients and I am sure there are many colleagues who find themselves in the shielded category that share this sentiment. It would be much appreciated, therefore, if the CDO, NHS England or the BDA could consider cases of dentists, and other healthcare professionals, such as myself and issue further guidance as to when it is safe to return to practice.

K. Oberai, Twickenham, UK

https://doi.org/10.1038/s41415-020-1795-0

\section{Paediatric emergencies}

Sir, whilst there has been a significant decrease in attendance to Emergency Departments during the COVID-19 pandemic, ${ }^{1}$ the Paediatric Emergency Department (PED) at Alder Hey Children's Hospital has observed little change in the number of dental attendances. We compared the characteristics of attendances in March, April 2020 to the same months in 2019 with three key findings to consider in the months ahead, as we resume dental services.

Firstly, the number of children attending the PED with traumatic dental injuries (TDIs) increased in the COVID-19 affected period. This may be due to decreased availability of primary care dental services in the region. Additionally, with more children undertaking exercise and activities at home with school closures, more accidental injuries may occur. It is also important to consider the possibility of TDIs related to non-accidental injury during lockdown, with children living in violent or dysfunctional families having limited access to safe spaces, friends, teachers and support outside the home in this period, leading to an increase in domestic violence and abuse. ${ }^{2}$ On presentation, it is important to take a thorough history and examination, identifying any discrepancies which may raise suspicion. ${ }^{3,4}$ The BSPD have published excellent guidelines to aid us with the management and follow up of TDIs during the COVID-19 pandemic. $^{5}$

Secondly, we found that the number of admitted patients decreased, suggesting that conditions of lower severity were presenting to the PED. This may also be attributable to a lack of alternatively available dental services.

Finally, as a result of the pandemic, thousands of children nationally have experienced cancellation of dental general anaesthetics (GA), with cases being prioritised as per national guidelines. ${ }^{6}$ Subsequently, thousands of children have been left in pain, taking regular analgesics and sometimes requiring multiple courses of antibiotics. Indeed, the number of children receiving repeat courses of antibiotics from the PED increased in the COVID affected period.

With GDPs reopening and reaching a 'new normal', the emergency management of TDIs and children in pain should be considered a priority in paediatric primary care triage to reduce the subsequent burden on tertiary services, which carries increased expense to the NHS.

\section{N. Gallichan, C. Heggie, S. Lee, S. Messahel, S. Albadri, Liverpool, UK}

\section{References}

1. NHS England, NHS Improvement. A\&E Attendances and Emergency Admissions April 2020 Statistical Commentary. 2020.

2. World Health Organisation, Joint Leaders' statement. Violence against children: a hidden crisis of the COVID 19 pandemic 2020. Available at: https://www.who.int/ news-room/detail/08-04-2020-joint-leader-s-statement--violence-against-children-a-hidden-crisis-of-the-covid19-pandemic (accessed June 2020).

3. Cairns A, Mok J Y, Welbury R. Injuries to the head, face, mouth and neck in physically abused children in a community setting. Int I Paediatr Dent 2005; 15: 310-318.

4. Becker D B, Needleman H L, Kotelchuck M. Child abuse and dentistry: orofacial trauma and its recognition by dentists. J Am Dent Assoc 1978; 97: 24-28.
5. British Society of Paediatric Dentistry. Trauma Guidelines for Primary/Permanent Dentition. Available at: https:// www.bspd.co.uk/resources (accessed June 2020)

6. Royal College of Surgeons England. Recommendations for Paediatric Dentistry during COVID-19 pandemic. RCS, 2020. Available at: https://www.rcseng.ac.uk/-/media/ files/rcs/fds/guidelines/paediatric-dentistry-covid19.pdf (accessed June 2020).

https://doi.org/10.1038/s41415-020-1796-z

\section{Child-friendly PPE}

Sir, eye contact and verbal communication with a child play a key role in nonpharmacological behaviour management. During this pandemic, when I visited a patient for dental urgency, I addressed the child in a conventional PPE kit abiding by the disinfection and safety protocols. The child was completely surprised and frightened and was hesitant in allowing examination. So I spent a couple of hours tweaking the conventional PPE kit with a child-friendly touch that included incorporation of famous cartoon characters and emoticons to make the child happy (Fig. 1). I performed this in a sterile environment following the sanitisation protocols. Next time I entered the treatment room in the customised PPE kit there was an overwhelming response from the child who underwent the treatment procedure with no sign of anxiety or fear. In addition, the



